\section{To understand the origin of life we must first understand the role of normativity}

\author{
Tom Froese \\ tom.froese@oist.jp \\ Embodied Cognitive Science Unit \\ Okinawa Institute of Science and Technology Graduate University \\ Onna-son, Okinawa, Japan
}

\begin{abstract}
Deacon develops a minimal model of a nonparasitic virus to explore how nucleotide sequences came to be characterized by a code-like informational at the origin of life. The model serves to problematize the concept of biological normativity because it highlights two common yet typically implicit assumptions: (1) that life could consist as an inert form, were it not for extrinsic sources of physical instability, and (2) that life could have originated as a singular self-contained individual. I propose that the origin of life, the genetic code, and biological normativity more generally, lead us to reject this passive individualism.
\end{abstract}

Keywords: autopoiesis, enactive cognitive science, embodied cognition, genetic code, artificial life, synthetic biology

Deacon (2021) addresses the question of how complex organic molecules, such as the nucleotide sequences that make up RNA and DNA, first came to be characterized by a codelike informational codependence in the context of the origin of life. This return to the origin of life is a strategic move by Deacon, which serves the purpose of developing a minimal model of a possible physical implementation of what he calls, following the tradition of biosemiotics, the "interpretation" of sign vehicles. Deacon's approach aligns closely with the enactive approach to autopoiesis and adaptivity - living is sense-making at its core and from its very start (Thompson, 2007). Yet there are also some productive differences between these approaches that are worth exploring in more detail.

The most fundamental difference lies in distinct criteria of what would count as a successful explanation. Deacon adheres to a narrow form of naturalism, in which the only permissible explanatory factors are those captured by the natural sciences. The enactive approach tends to adopt a more relaxed form of naturalism, in which the subjective side of life can also make a difference in its own right, including normatively, to how its behavior unfolds (Hanna \& Maiese, 2009). It is this qualitative transition from non-normative to normative, which is a transition that goes beyond mere chemistry, that is at the root of why the origin of life remains such an intractable problem. Researchers in artificial life and synthetic biology pursuing the creation of life from scratch are faced by a profound challenge: to create a novel system that is sensitive to a normativity that is not derived, like that of all our other artifacts, but is intrinsic to that system (Froese \& Taguchi, 2019).

How did intrinsic normativity originate in nature? An important clue can be found in the fact that living systems must continually do work - metabolically and regulatorily - to even exist (Kauffman, 2000). In contrast to events in purely physical systems, which merely happen 
without any sense of their consequences, activity in biological systems essentially involves doing things for a purpose, i.e., trying or striving, which implies a normative condition for their consequences, especially for the possibility of continued existence. The doings of the living ultimately matter to the living because of mortality: the possibility of success or failure is intrinsically related to possibility of life or death (Froese, 2017). Indifference is not option.

Thus, the origin of life does not consist only in an increase in the complexity of chemical organization; it is also the point at which biological normativity for the first time comes to play a role on our planet, and possibly in the whole universe. To do justice to this qualitative transition it will be necessary, first, to make conceptual room in the natural sciences such that normativity can make a difference on its own terms, beyond the determinations derived from its physical implementation. Second, there is still the unresolved challenge to explain how this initial form of life, with its basic normativity of doing for the sake of being, increases in complexity in an open-ended manner. For this latter part, it will become crucial to explain the origin of the genetic code.

In what follows I will mainly focus on the more philosophical aspects of the first issue, and I will finish by sketching some of the implementation aspects of the second issue.

\section{On the basis and efficacy of normativity}

Deacon positions his model as a counterpoint to classic modern biology, as epitomized by Dawkins" "selfish gene" and the "RNA World" scenario of the origin of life, which reduces the process of interpretation of information to mere molecular replication. His ambition is to develop an alternative to this reductionist position by making it intelligible how a molecular structure, which in itself is just a physical pattern, could have informational content, i.e., be about something. ${ }^{1}$ And this in turn leads Deacon to develop his model as a minimal physical implementation of the process of interpretation.

The core of Deacon's "autogen" model is the self-organizing dynamics of two codependent processes, reciprocal catalysis and capsid self-assembly. It is therefore a variation on the familiar theme of bounded self-production, which includes a long and venerable tradition of proposals at various levels of abstraction, including "autopoiesis" (Varela, Maturana, \& Uribe, 1974), "autocatakinetic closure" (Swenson, 2000), "autonomous agent" (Kauffman, 2000), "basic autonomy" (Ruiz-Mirazo \& Moreno, 2004), and the "metabolism-repair (M-R) system" (Rosen, 1991). Luisi (2006) provides an extensive review of such "metabolism-first" approaches to the origin of life, including of the many efforts to realize their physical implementation. It is a pity that Deacon does not engage more with this extant literature, because it remains unclear in which precise respects his "autogen" model is an advance over the state of the art in this field with respect to grounding normativity.

What is innovative about Deacon's proposal is to cast bounded self-production in terms of Peirce's theory of signs. He provides a step-by-step semiotic theory of the normativity

\footnotetext{
${ }^{1}$ I side with Hutto and Myin (2013) that the states of basic minds, including of minimal lifeforms, are strictly speaking contentless because they are not "about" anything in a strong semantic sense, e.g. involving truth conditions. But we all agree that basic minds can nevertheless be intentionally directed at aspects of the world, and this is sufficient common ground for the current discussion.
} 
involved at the transition from the origin of life to the origin of the genetic code. Another conceptual novelty of Deacon's proposal is that he explicitly defines his model of bounded self-production as a kind of non-parasitic virus. This has the virtue of highlighting that, at the system level, self-producing systems can be characterized by an inert form, which reveals a hidden tension with Deacon's appeal to active adaption as the basis of normativity.

Deacon is not alone with this problem. The identification of the default state with an inert state is also a feature of famous cybernetic models of adaptation, including ultrastability and classic autopoiesis (Froese \& Stewart, 2010). In Deacon's model, the initial state even consists in a stop of all processes inside a static capsid with an "inert form". Hence, catalysis and self-assembly are only temporary reactions to an "extrinsic disruption" that led to the loss of boundary integrity. At an abstract level, this model belongs to the class of generic physical systems that when pushed out of stable equilibrium by independent factors will mount counteracting forces that allow it to reconverge. It can therefore be doubted whether this kind of extrinsically caused reactivity is sufficient for Deacon to attribute to the system any kind of intrinsic activity. If this shift from extrinsic to intrinsic cannot be secured, the model would be lacking the most essential ingredient on which Deacon's semiotic notion of normativity depends.

As a comparative case, consider Deacon's rejection of selectionist accounts as inadequate for grounding active adaptation and normativity, given that "the 'external' environment does all the work". The question is therefore how to characterize the condition of the living such that it is not subject to similar criticisms. The three-decade history of the enactive approach reflects the search for an answer to this question, which has resulted in a more refined understanding of intrinsic activity.

The idea that boundary repair could serve as the basis for normativity goes back to the very initial formulations of the enactive approach. Consider Varela's proposal that autopoiesis can serve as the basis for a biology of "intentionality". It is precisely the concept of a "breakdown" of an otherwise static identity, and the system's initiation of an adaptive response, which motivates the claim that a meaningful world shows up for the organism:

"The source for this world-making is always the breakdowns in autopoiesis, be they minor, like changes in concentration of some metabolite, or major, like disruption of the boundary. Due to the nature of autopoiesis itself - illustrated in the membrane repair of the minimal simulated example above - every breakdown can be seen as the initiation of an action on what is missing on the part of the system so that identity might be maintained." (Varela, 1992)

Like Deacon's model, Varela's initial proposal can therefore also be criticized as insufficient for active adaptation. However, since then several conceptual advances have been made, which can be briefly summarized as follows.

First, there was an explicit recognition that a proper grounding of normativity requires that the source of the system's vulnerability to disruption has to be expanded from the extrinsic environment to the intrinsic constitution of the living, which came to be referred to as their "precariousness" (Weber \& Varela, 2002). Second, it was recognized that a graded and 
differentiated perspective of sense-making required a system capable of more states than self and non-self. In contrast to Deacon's claim that this "zeroth" level of semiosis would include "a sign of non self", not even this is given: everything would have an absolutely positive sense (as long as "self" exists) or absolutely no sense at all (no "self" exists). One proposal for grounding a range of normativity was that the system is sensitive to the relative amount of effort that is required for its adaptive response to keep the state of its essential variables away from its boundary of viability (Di Paolo, 2005). Third, adaptivity was rooted in the instability of bounded self-production as such. There is an irreducible tension in the system between satisfying the needs of self-individuation or boundedness (being as closed as possible to the environment) and the needs of self-production (being as open as possible to the environment). This enables us to define active adaptation as the spontaneous change of system configurations whereby the mutually exclusive conditions of bounded selfproduction are iteratively and always only partially resolved (Di Paolo, 2018). A key move, therefore, is to characterize the default state of the living system as intrinsically unstable, which at the same time enables us to characterize it as intrinsically active.

This still leaves open the bigger mystery of how these spontaneous reconfigurations of the system are subject to normative regulation as such. How does the value associated with satisfying normative conditions enter this scenario? A clue is that the irreducible partialness of each of the adopted configurations points to an essential incompleteness at the core of the living, which also resonates with Deacon's (2012) own emphasis on the incompleteness of nature when we probe it for phenomena of intentionality. Pushing these ideas further, we could start to view the role of normativity not as a specific cause, which is always in danger of collapsing into just another physical determination, but rather as precisely the relative absence or bracketing of physical determination (Froese \& Taguchi, 2019). The physical indeterminacy that would be associated with normative dependencies could then be measured in terms of uncertainty measures like entropy. Intriguingly, this suggests a new way of considering the link between the origin of life and the principle of maximum entropy production, which deserve to be explored in more detail elsewhere.

There are additional implications for placing instability and incompleteness at the core of the living: once this dynamic interplay between openness and closedness becomes part of the system's own regulatory process, the concept of a rigid boundary, like a capsid, is no longer an appropriate model. A more permeable boundary, like a chemical gradient, may be a more suitable starting point for this active system-environment interaction (Froese, Virgo, \& Ikegami, 2014). But such fluidity brings along its own challenges of adaptability and evolvability, which depends on more solid molecular structures, so perhaps life originated on the surface of droplets (Sharov, 2016). From this more dynamic starting point, a key step in the increase of system complexity will have to do with increasing state-dependence of internal and interactive behavior. In line with Deacon's proposal, this suggests that the genetic system first started as a stable memory system, which only later became involved in transmission across generations. But, as he also recognizes, this still falls short of explaining the origins of the genetic code. There is a missing mechanism that can account for the transition from an individual's memory system to a cross-generational genetic system.

A possible mechanism for the origin of the genetic code that is consistent with Deacon's proposal is based on horizontal gene transfer. Simulation models show that even molecular 
sequences with an initially arbitrary association between genes and proteins spontaneously take on a code-like structure, as long as they are iteratively passed between systems that only partially acquire its correlational mapping, similar to the iterated learning paradigm in language evolution (Froese, Campos, Fujishima, Kiga, \& Virgo, 2018). This iterative approach seems to be in tension with the fitness cost of changing the mappings of the genetic code, which is why some favor scenarios wherein a static code originates as a whole like a frozen accident. But the highly ordered nature of the genetic code makes that scenario highly unlikely, and the worry about costly changes could be addressed by envisioning a scenario initially involving non-deleterious changes, including an expansion of the code by increasing the number of nucleotide "letters" (Frank \& Froese, 2018).

This model of the origin of the genetic code is suggestive, but it leaves several key questions unsolved. First, it requires a spatially contained population of proto-living systems at a relatively advanced stage of complexity, which runs counter to Deacon's claim that life "arose by accident". However, there are compelling pre-biological mechanisms that could account for their "design", such as population-level proto-cell optimization in the wet-dry cycles of Archaean ponds (Damer, 2016). Second, the model leaves unclear the origin of horizontal gene transfer. An intriguing possibility is suggested by Deacon's development of Dyson's two-stage origin of the genetic code, according to which nucleotides first stored and transported chemical energy in nonreactive forms, and these molecules only later acquired code-like properties. Horizontal gene transfer can then be conceived as originating in the context of a population of proto-cells participating in a network of energy exchange.

\section{Conclusions}

More work clearly needs to be done to flesh out all these ideas. Yet, taken together, it is evident that they are pointing in a very different direction than the passive individualism implied by the "autogen" model. Rather than grounding normativity by starting from a static, solitary, and self-contained system, we are left with a sense of the fundamental role of intrinsic instability, openness, and interactivity. This suggests that we should consider an altogether different starting point. For instance, a more suitable proposal for the origin of life could be in terms of the emergence of an organic geosphere: a global network of chemical reactions that was particularly suited to reducing the energetic gradients that had been produced by the pre-organic geosphere (Smith \& Morowitz, 2016). This leads to a final speculation: perhaps zero-level normativity first originated along with this whole biosphere, which maximized energy flow via self-production, and it only later complexified in terms of individual perspectives when self-production became partially bounded.

\section{References:}

Damer, B. (2016). A field trip to the Archaean in search of Darwin's warm little pond. Life, 6(2), 21. doi:10.3390/life6020021

Deacon, T. W. (2012). Incomplete Nature: How Mind Emerged from Matter. New York, NY: W. W. Norton \& Company.

Deacon, T. W. (2021). How molecules became signs. Biosemiotics. Advance online publication. https://doi.org/DOI:10.1007/s12304-021-09453-9. 
232

233

234

235

236

237

238

239

240

241

242

243

244

245

246

247

248

249

250

251

252

253

254

255

256

257

258

259

260

261

262

263

264

265

266

267

268

269

270

271

272

273

274

275

276

277

278
Di Paolo, E. A. (2005). Autopoiesis, adaptivity, teleology, agency. Phenomenology and the Cognitive Sciences, 4(4), 429-452.

Di Paolo, E. A. (2018). The enactive conception of life. In A. Newell, S. Gallagher, \& L. De Bruin (Eds.), The Oxford Handbook of 4E Cognition (pp. 71-94). Oxford, UK: Oxford University Press.

Frank, A., \& Froese, T. (2018). The Standard Genetic Code can Evolve from a Two-Letter GC Code Without Information Loss or Costly Reassignments. Origins of Life and Evolution of Biospheres, 48(2), 259-272. doi:10.1007/s11084-018-9559-4

Froese, T. (2017). Life is precious because it is precarious: Individuality, mortality, and the problem of meaning. In G. Dodig-Crnkovic \& R. Giovagnoli (Eds.), Representation and Reality in Humans, Other Living Organisms and Intelligent Machines (pp. 30-55). Switzerland: Springer.

Froese, T., Campos, J. I., Fujishima, K., Kiga, D., \& Virgo, N. (2018). Horizontal transfer of code fragments between protocells can explain the origins of the genetic code without vertical descent. Scientific Reports, 8, 3532. doi:10.1038/s41598-018-21973$y$

Froese, T., \& Stewart, J. (2010). Life after Ashby: Ultrastability and the autopoietic foundations of biological individuality. Cybernetics \& Human Knowing, 17(4), 83-106.

Froese, T., \& Taguchi, S. (2019). The problem of meaning in Al and robotics: Still with us after all these years. Philosophies, 4, 14. doi:10.3390/philosophies4020014

Froese, T., Virgo, N., \& Ikegami, T. (2014). Motility at the origin of life: Its characterization and a model. Artificial Life, 20(1), 55-76.

Hanna, R., \& Maiese, M. (2009). Embodied Minds in Action. Oxford: Oxford University Press. Hutto, D. D., \& Myin, E. (2013). Radicalizing Enactivism: Basic Minds without Content. Cambridge, MA: The MIT Press.

Kauffman, S. A. (2000). Investigations. New York, NY: Oxford University Press.

Luisi, P. L. (2006). The Emergence of Life: From Chemical Origins to Synthetic Biology. Cambridge: Cambridge University Press.

Rosen, R. (1991). Life Itself: A Comprehensive Inquiry Into the Nature, Origin and Fabrication of Life. New York, NY: Columbia University Press.

Ruiz-Mirazo, K., \& Moreno, A. (2004). Basic autonomy as a fundamental step in the synthesis of life. Artificial Life, 10(3), 235-259.

Sharov, A. A. (2016). Coenzyme world model of the origin of life. BioSystems, 144, 8-17.

Smith, E., \& Morowitz, H. (2016). The Origin and Nature of Life on Earth: The Emergence of the Fourth Geosphere. Cambridge, UK: Cambridge University Press.

Swenson, R. (2000). Spontaneous order, autocatakinetic closure, and the development of space-time. Annals of the New York Academy of Sciences, 901, 311-319.

Thompson, E. (2007). Mind in Life: Biology, Phenomenology, and the Sciences of Mind. Cambridge, MA: Harvard University Press.

Varela, F. J. (1992). Autopoiesis and a biology of intentionality. In B. McMullin \& N. Murphy (Eds.), Autopoiesis and Perception: A Workshop with ESPRIT BRA 3352 (Addendum) (pp. 4-14). Dublin, Ireland: Dublin City University.

Varela, F. J., Maturana, H. R., \& Uribe, R. (1974). Autopoiesis: The organization of living systems, its characterization and a model. BioSystems, 5, 187-196.

Weber, A., \& Varela, F. J. (2002). Life after Kant: Natural purposes and the autopoietic foundations of biological individuality. Phenomenology and the Cognitive Sciences, 1(2), 97-125. 
Commentary on the target article by Terrence Deacon in Biosemiotics

279

280

Conflict of interest statement: The author has declared no conflict of interest.

281

282 